\title{
EFT validity issues in Vector Boson Scattering processes
}

\author{
Michał Szleper, ${ }^{a, *}$ Geetanjali Chaudhary, ${ }^{b}$ Jan Kalinowski, ${ }^{c}$ Manjit Kaur, ${ }^{b}$ Paweł \\ Kozów, ${ }^{d}$ Stefan Pokorski, ${ }^{c}$ Kaur Sandeep ${ }^{b}$ and Sławomir Tkaczyk ${ }^{e}$ \\ ${ }^{a}$ National Center for Nuclear Research, \\ ul. Pasteura 7, 02-093 Warszawa, Poland \\ ${ }^{b}$ Panjab University, \\ Chandigarh-160014, India \\ ${ }^{c}$ Faculty of Physics, University of Warsaw, \\ ul. Pasteura 5, 02-093 Warszawa, Poland \\ ${ }^{d}$ CAFPE and Departamento de Física Teórica y del Cosmos, Universidad de Granada, \\ Campus de Fuentenueva, 18071 Granada, Spain \\ ${ }^{e}$ Fermi National Accelator Laboratory, \\ Batavia, IL 60510, USA \\ E-mail: Michal.Szleper@ncbj.gov.pl, Geetanjali.Chaudhary@cern.ch, \\ Jan.Kalinowski@fuw.edu.pl, Manjit.Kaur@cern.ch, Pawel.Kozow@fuw.edu.pl, \\ Stefan.Pokorski@fuw.edu.pl, Sandeep.Kaur.Hundal@cern.ch, tka@fnal.gov
}

\begin{abstract}
Vector Boson Scattering (VBS) processes are regarded as the best lab to study the $V V V V$ quartic couplings, where $V=W, Z$. Such studies are carried in the framework of Effective Field Theories (EFT), but the EFT formalism is often not used in a fully consistent way. We discuss the limitations of the EFT approach to describe New Physics effects in VBS data. We argue that the "clipping" technique is the most theory-motivated way to do data analysis in the EFT language and discuss first results from an analysis of CMS Run 2 data on the $W Z$ and same-sign $W W$ process, with and without "clipping" implemented.
\end{abstract}

The Eighth Annual Conference on Large Hadron Collider Physics-LHCP2020

25-30 May, 2020

online

\footnotetext{
${ }^{*}$ Speaker
} 


\section{Introduction}

Study of VBS processes as an indirect search for physics Beyond the Standard Model (BSM) has already a respectable tradition in both experimental and theoretical literature [1]. The point of focus of such studies is usually the $V V V V$ quartic coupling whose potential deviations from Standard Model (SM) predictions can be parameterized by dimension- 8 operators in the EFT expansion.

It is well known that every dimension- 8 operator induces amplitude growth which ultimately leads to violation of the unitarity condition at some energy scale. In the range of Wilson coefficients we can currently probe at the LHC, unitarity violation occurs well within the accessible range of the $V V$ mass, hence the issue has to be addressed in some way in data analysis. Applying the EFT expansion as if it was valid in the whole measured kinematic range, which means disregarding the unitarity limits, also means giving up on the physical interpretation of the Wilson coefficients. Various techniques of amplitude unitarization are aimed at finding the maximum possible signal that can be associated to a given Wilson coefficient. However, the results are largely driven by the details of the unitarization model one assumes (recently [2]). An ideal procedure to deal with the unitarity issue should retain model independence of the EFT on the one hand, while watching strictly the EFT range of validity on the other.

\section{Principles of EFT validity}

Parameter $\Lambda$ in the EFT expansion bounds the actual range of validity of the formalism. The unitarity condition defines its maximum possible value, but the actual value is unknown a priori and can only be deduced by experiment. For this reason, any real EFT data analysis needs to be carried in at least two dimensions: one or more Wilson coefficient $f$, plus $\Lambda$ as an independent parameter which determines the scale up to which we have applied the EFT expansion.

By construction, $\Lambda$ is a single value for a given operator, hence it is the same for all the individual helicity combination amplitudes, as well as for different processes that are governed by the same operator or set of operators. The lowest unitarity limit that is relevant to a given operator determines the maxium value of $\Lambda$ which is physically plausible. Consequently, for most dimension8 operators: $O_{S 1}, O_{T 0}, O_{T 1}$ (positive $f$ ), $O_{T 2}, O_{M 0}, O_{M 1}$ and $O_{M 7}$, the maximum physical values of $\Lambda$ for the same-sign $W W$ process are dictated by unitarity limits in the opposite-sign $W W$ process. This is because the latter are always lower (especially for $M$ operators) than the respective unitarity limits in the same-sign process. Similarly, the unitarity limits that are relevant for the $W Z \rightarrow W Z$ process are, for most dimension- 8 operators, driven by the $W W \rightarrow Z Z$ process.

\section{EFT validity in BSM discovery}

In processes where the $V V$ invariant mass cannot be determined experimentally, like the leptonic $W W$ process (also $W Z$ and $W V$, unless a technique is worked out to reliably solve the quadratic ambiguity in the kinematics of the missing neutrino), the measured signal is a priori the sum of two kinematic regions: below and above $\Lambda$, where in the latter the EFT cannot be applied. The EFT can provide a successful description of the data only so long as the bulk of the signal originates from the former rather than the latter region. This observation led to the concept of 
"EFT triangles", first introduced in Ref. [3], i.e., the regions in the two-dimensional $(f, \Lambda)$ space where this criterion is fulfilled and at the same time BSM effects are detectable (see Fig. 1 right). The energy dependence of the $W^{+} W^{+} \rightarrow W^{+} W^{+}$process with an anomalous quartic coupling (see Fig. 1 left) reveals that sizeable deviations from the SM are generally confined to a narrow region just before the unitarity limit [4]. For this reason, in the leptonic same-sign $W W$ process, the "EFT triangles" for all the individual dimension- 8 operators are tiny or vanishing, both in the SMEFT formalism [3] and in the HEFT formalism [5].
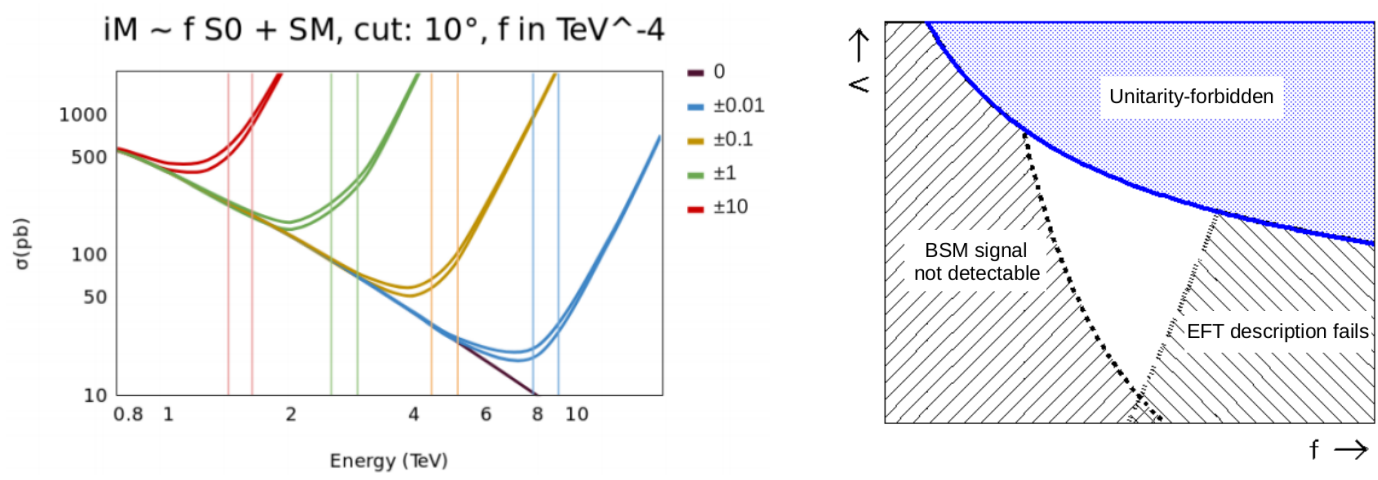

Figure 1: Left: cross section for the $W^{+} W^{+} \rightarrow W^{+} W^{+}$scattering process (on shell approximation) as a function of the center of mass energy, in the SM and for different values of $f_{S 0}$. The corresponding unitarity limits are marked with vertical lines. Right: conceptual representation of an "EFT triangle" for a single Wilson coefficient $f$ : only in the central white region of the $(f, \Lambda)$ plane correct EFT description of a hypothetical BSM signal is possible.

\section{EFT validity vs. setting limits on BSM}

In order to apply the EFT formalism in a physically interpretable and model independent way, the kinematic region where the EFT is not applicable should be omitted. This involves a high mass cutoff at a preselected value of $\Lambda$. For a process where the $V V$ invariant mass is not measured, such cutoff is possible only on simulation. To compensate for the fact that real data may have an additional contribution from above $\Lambda$, the SM high mass tail is added to the simulation. This is the basis of the "clipping" method. Computation of BSM signal from solely the EFT validity region corresponds to the most conservative model independent limits on the Wilson coefficients.

In a recent paper, the CMS collaboration has produced a new analysis of the fully leptonic same-sign $W W$ and $W Z$ processes, based on data collected during Run 2 of the LHC, corresponding to a total of $137 \mathrm{fb}^{-1}$ [6]. In this analysis, partial "clipping" was applied to calculate the limits on the individual dimension- 8 operators, namely, simulated samples were "clipped" at the respective unitarity limits in order to compute the theory predictions. Typical unitarity limits in the $f$ ranges we are presently sensitive to are close to $1.5 \mathrm{TeV}$. This corresponds to the removal (or SM-reweighting) of up to $50 \%$ of the generated $W Z$ events and up to $80 \%$ of the generated $W W$ events. Table 1 shows the obtained (observed) 95\% CL limits on all the relevant dimension-8 operators, for $W^{ \pm} W^{ \pm}$, $W Z$ and combined, with and without "clipping". As can be seen, the "clipped" results are looser by typically around a factor $4-5$ than the non-"clipped". 


\begin{tabular}{|c|c|c|c|c|c|c|}
\hline & $\begin{array}{c}W^{ \pm} W^{ \pm} \\
\text {Not clipped } \\
\left(\mathrm{TeV}^{-4}\right)\end{array}$ & $\begin{array}{c}W Z \\
\text { Not clipped } \\
\left(\mathrm{TeV}^{-4}\right)\end{array}$ & $\begin{array}{c}\text { Combined } \\
\text { Not clipped } \\
\left(\mathrm{TeV}^{-4}\right)\end{array}$ & $\begin{array}{c}W^{ \pm} W^{ \pm} \\
\mathrm{Clipped} \\
\left(\mathrm{TeV}^{-4}\right)\end{array}$ & $\begin{array}{c}W Z \\
\text { Clipped } \\
\left(\mathrm{TeV}^{-4}\right)\end{array}$ & $\begin{array}{c}\text { Combined } \\
\text { Clipped } \\
\left(\mathrm{TeV}^{-4}\right)\end{array}$ \\
\hline$f_{T 0}$ & {$[-0.28,0.31]$} & {$[-0.62,0.65]$} & {$[-0.25,0.28]$} & {$[-1.5,2.3]$} & {$[-1.6,1.9]$} & {$[-1.1,1.6]$} \\
$f_{T 1}$ & {$[-0.12,0.15]$} & {$[-0.37,0.41]$} & {$[-0.12,0.14]$} & {$[-0.81,1.2]$} & {$[-1.3,1.5]$} & {$[-0.69,0.97]$} \\
$f_{T 2}$ & {$[-0.38,0.50]$} & {$[-1.0,1.3]$} & {$[-0.35,0.48]$} & {$[-2.1,4.4]$} & {$[-2.7,3.4]$} & {$[-1.69,3.1]$} \\
$f_{M 0}$ & {$[-3.0,3.2]$} & {$[-5.8,5.8]$} & {$[-2.7,2.9]$} & {$[-13,16]$} & {$[-16,16]$} & {$[-11,12]$} \\
$f_{M 1}$ & {$[-4.7,4.7]$} & {$[-8.2,8.3]$} & {$[-4.1,4.2]$} & {$[-20,19]$} & {$[-19,20]$} & {$[-15,14]$} \\
$f_{M 7}$ & {$[-6.7,7.0]$} & {$[-10,10]$} & {$[-5.7,6.0]$} & {$[-22,24]$} & {$[-22,22]$} & {$[-16,18]$} \\
$f_{S 0}$ & {$[-6.0,6.4]$} & {$[-19,19]$} & {$[-5.7,6.1]$} & {$[-35,36]$} & {$[-83,85]$} & {$[-34,35]$} \\
$f_{S 1}$ & {$[-18,19]$} & {$[-30,30]$} & {$[-16,17]$} & {$[-100,120]$} & {$[-110,110]$} & {$[-86,99]$} \\
\hline
\end{tabular}

Table 1: 95\% CL limits on dimension-8 operators from a CMS analysis of Run 2 data, for $W^{ \pm} W^{ \pm}$, WZ and combined. Columns 2-4 correspond to standard, non-"clipped" results, columns 5-7 correspond to "clipped" results. Data from Ref. [6].

In the full "clipping" method one calculates limits on $f$ as a function of the assumed value of $\Lambda$, the latter ranging between the lowest accessible value and the unitarity limit. Comparison of the resulting experimental curves with the purely theoretical curves induced by the unitarity limit (see blue curve in Fig. 1 right) will make it straighforward to find whether, and in what ranges of $\Lambda$, experiment can already place physically meaningful limits on dimension- 8 operators. Such analysis is currently in progress and results are expected soon.

\section{References}

[1] A list of recently published VBS-related work is available at the VBSCan website https://twiki.cern.ch/twiki/bin/view/VBSCan/VBSCanPublications.

[2] C. Garcia-Garcia, M. J. Herrero, R. A. Morales, Unitarization effects in EFT predictions of WZ scattering at the LHC, Phys. Rev. D 100 (2019) 096003 [arXiv: 1907.06668 [hep-ph]].

[3] J. Kalinowski, P. Kozów, S. Pokorski, J. Rosiek, M. Szleper, S. Tkaczyk, Same-sign WW scattering at the LHC: can we discover BSM effects before discovering new states?, Eur. Phys. J. C 78 (2018) 403 [arXiv: 1802.02366 [hep-ph]].

[4] G. Chaudhary, J. Kalinowski, M. Kaur, P. Kozów, K. Sandeep, M. Szleper, S. Tkaczyk, EFT triangles in the same-sign WW scattering process at the HL-LHC and HE-LHC, Eur. Phys. J. C 80 (2020) 181 [arXiv: 1906.10769 [hep-ph]].

[5] P. Kozow, L. Merlo, S. Pokorski, M. Szleper, Same-sign WW Scattering in the HEFT: Discoverability vs. EFT Validity, JHEP 07 (2019) 021 [arXiv: 1905.03354 [hep-ph]].

[6] CMS Collaboration, Measurements of production cross sections of $W Z$ and same-sign $W W$ boson pairs in association with two jets in proton-proton collisions at $\sqrt{s}=13 \mathrm{TeV}$, Phys. Lett. $B$ 809 (2020) 135710 [arXiv: 2005.01173 [hep-ex]]. 